\title{
Pensare in numeri e usare i numeri per comunicare il rischio
}

\author{
Giulio Marcon ', Emanuela Barbiero ${ }^{2}$ \\ Professore a contratto di Management Clinico, Università di Padova \\ 2 Professore a contratto di Infermieristica in area specialistica, Università di Padova e Università di Udine
}

\begin{abstract}
Bad presentation of the risks associated with a medical intervention can lead to patients making poor decisions on treatment. Some ways in communicating clinical risk are particularly confusing, expecially when physicians use conditional probabilities (such as sensitivity and specificity), and relative risks. However, the inability to understand statistical information is not a mental deficiency of doctors or patients but is largely due to the poor presentation of the information. This articles gives some advices to improve an adequate communication of statistical information, so that patients can make well informed decisions.
\end{abstract}

Keywords: communicating clinical risk, statistical information, doctor-patient communication

Think in number and use the number in clinical risk communication

Pratica Medica \& Aspetti Legali 2008; 2(3): 137-142

Il consenso informato è un elemento chiave per la sicurezza del paziente. Perché il consenso sia valido, è necessario che il/la paziente comprenda i rischi e i benefici dell'intervento proposto [1]. Questo implica che i professionisti sanitari siano in grado di comunicare i rischi; ciò significa che $\mathrm{i}$ medici non solo devono avere chiari i rischi connessi ad un intervento, ma devono essere anche in grado di comunicarli ai propri pazienti in modo comprensibile e chiaro. Inoltre, nel caso di interventi che comportano un grado di rischio sconosciuto o poco conosciuto, le incertezze dell'intervento proposto devono essere segnalate al/alla paziente.

Sfortunatamente, la formazione relativa alla valutazione dei rischi non fa parte del normale curriculum formativo dei professionisti sanitari e raramente questa formazione viene offerta nel corso della pratica clinica.

Generalmente il rischio viene presentato in termini numerici, ma non vi è, nel curriculum formativo dei professionisti sanitari, una preparazione specifica che addestri a pensare in numeri e a usare $\mathrm{i}$ numeri per quantificare il rischio.
Normalmente, ad esempio non distinguiamo tra rischio personale (ad esempio, il rischio di avere un bambino con una malattia autosomica recessiva è di 1:4 se entrambi i geni sono portatori) e rischio empirico (rischio derivato dagli studi su popolazione: ad esempio 1/200 donne che vengono sottoposte ad amniocentesi possono avere come conseguenza dell'amniocentesi un aborto spontaneo causato dalla procedura).

Inoltre è possibile sbagliare nell'applicare le informazioni relative al rischio al contesto adatto. Ciò avviene ad esempio se confrontiamo il rischio di aborto legato alla procedura di un'amniocentesi eseguita alla $17^{\text {a }}$ settimana con una biopsia dei villi coriali eseguita alla $11^{\text {a }}$ settimana, senza tenere in conto del rischio di aborto spontaneo e le rispettive età gestazionali.

Inoltre, non siamo sempre al corrente dei fattori personali che influenzano l'accettazione o meno dei rischi da parte dello specifico paziente, il che rende difficile capire le scelte dei malati.

A causa di questi limiti, spesso non siamo capaci di valutare e di comunicare correttamente ai pa- 
zienti il rischio, non consentendo al paziente di prendere la decisione più informata.

Sia i professionisti sanitari che i pazienti hanno la necessità di saper esprimere e comunicare il rischio. In un suo testo [2], Gerd Gigerenzer osserva come alcune culture abbiano una fame insaziabile di numeri, medie e indici numerici, mentre altre culture siano riluttanti ad esprimersi, nel campo dell'incertezza, in termini numerici.

Gigerenzer lamenta inoltre il fatto che, mentre è generalmente accettato che «il pubblico ha diritto ad avere informazioni», non vi è alcun consenso sul fatto che il pubblico abbia il diritto di avere queste informazioni in modo chiaro e non confondente. Egli chiede quindi con forza che le associazioni mediche, legali e di volontariato "sottoscrivano una politica etica che obblighi a chiarire i rischi in termini chiari e comprensibili».

Nel suo libro, Gigerenzer stigmatizza due tipi di ambiguità legate all'uso dei termini numerici:

- la comunicazione inefficiente sul rischio: la persona che comunica conosce il rischio ma non sa come comunicarlo agli altri in modo chiaro;

- il pensiero annebbiato: la persona che riceve informazioni sul rischio ne riconosce l'esistenza, ma non è in grado di trarre le conclusioni o le inferenze utili per prendere le decisioni.

Gigerenzer individua inoltre tre principali tipologie di comunicazione del rischio che tendono a generare una comunicazione errata:

- l'uso delle probabilità per un "evento singolo": «il rischio di morire per questo intervento è del $10 \%$;

- il "rischio relativo": «lei ha, rispetto alla norma, una probabilità di morire tre volte maggiore se decide di affrontare l'intervento proposto, rispetto al non fare niente; le propongo pertanto di non sottoporsi all'intervento e di continuare ad assumere i farmaci che sta assumendo»;

- le probabilità "condizionali”: «se si sottopone a questo intervento, ha il $90 \%$ di probabilità di sopravvivere», il che può essere confuso con «il $90 \%$ delle persone che sopravvivono ha subìto questo intervento».

Il modo nel quale viene contestualizzato il rischio ha una notevole importanza sulla sua percezione, e Gigerenzer ne fornisce un esempio illuminante correlato all'uso del test del DNA. Immaginiamo il caso di una persona che debba comparire in Tribunale in quanto sulla scena del crimine sono state trovate tracce di DNA corrispondenti al suo.

L'esperto può quindi affermare che «dato che la probabilità che si verifichi questo caso (ossia la corrispondenza tra DNA rilevato e quello del soggetto) è di 1 su 100.000, è difficile immaginare che il crimine sia stato commesso da una persona diversa dall'accusato».

Lesperto tuttavia può anche fornire una interpretazione differente che recita «di 100.000 persone, solo una ha un DNA uguale a quello del soggetto». Quest' affermazione può suonare come «in una città di 1.000 .000 di abitanti vi sono 10 abitanti il cui DNA è uguale al DNA indice».

Usando questo esempio, Gigerenzer mostra come le probabilità, il rischio relativo e le percentuali debbano essere evitate quando si comunicano i rischi. Al loro posto, vanno usati il rischio assoluto e le frequenze naturali. Ad esempio, se a una donna viene spiegato che un particolare trattamento può raddoppiare il rischio di insorgenza di neoplasia mammaria (rischio relativo), viene chiaramente gettata nel panico; se invece la spiegazione è data in termini di rischio assoluto (il trattamento aumenta il rischio di neoplasia mammaria da $1 / 1.000$ a $2 / 1.000$ delle donne che vi si sottopongono) sarà certamente più rassicurata. Si può porre la questione sotto un aspetto ancora migliore, chiarendo che il rischio di neoplasie mammarie causate dal trattamento è di 1/1.000 (frequenza), invece che dare il dato in termini percentuali. Una ragione per non usare le percentuali, specialmente quando il campione è piccolo, è detta "effetto Giuda": anche se un robusto $8 \%$ dei discepoli di Gesù lo aveva tradito, questo $8 \%$ era costituito tuttavia da una sola persona. Analogamente, un 26\% dell'aumento di neoplasie mammarie può sembrare allarmante, ma in termini reali può essere costituito dall'aumento di meno di un caso per 1.000 donne per anno.

\section{STRUTTURARE IL CONCETTO DI RISCHIO NELLA PRATICA CLINICA}

Nel lavoro sanitario di tutti i giorni, vengono emessi giudizi e prese decisioni che comportano la valutazione dei rischi connessi con giudizi e decisioni precedentemente definite. Le decisioni sono condizionate da una serie di fattori che comprendono il contesto nel quale vengono prese e la prospettiva psicologica del paziente. Ad esempio, vi può essere il rischio derivante dalla decisione di eseguire o meno un test diagnostico e vi può essere un rischio nella scelta di selezionare un esame particolare da una lista. Una valutazione sommaria dei fattori che sono in gioco nel rischio clinico dimostra che [3]:

- i rischi personali sono specifici per ogni paziente, mentre i medici hanno una percezione solo generale del rischio; 
- i rischi personali sono spesso sottostimati, e in particolare lo sono quelli derivanti da comportamenti pericolosi per la salute, poiché vengono percepiti come meno pericolosi delle malattie e dei rischi della popolazione generale;

- data la piccola differenza di percezione del rischio tra medici e pazienti, è spesso facile ottenere dal malato l'accettazione di un trattamento terapeutico proposto;

- esplicitare i rischi in modo chiaro aumenta la probabilità che le persone forniscano il loro consenso;

- la contestualizzazione delle informazioni relative al rischio diviene quindi un vantaggio per ottenere il consenso ad una terapia o a una procedura.

\section{IL CONTESTO}

Nela pratica medica quotidiana vengono continuamente prese decisioni e ed emessi giudizi che comportano la valutazione e l'accettazione di un rischio, ad esempio il rischio insito nella decisione di eseguire o meno una procedura diagnostica. A queste variabili si può inoltre sommare l'incertezza, ad esempio nell'interpretare i risultati delle indagini cliniche o nel decidere la terapia più adatta tra le varie opzioni disponibili.

Tenendo presente questa incertezza, in che modo si possono conciliare le informazioni derivanti dalla conoscenza biomedica da un lato e le informazioni derivanti dalla conoscenza clinica dall'altro in modo tale da raggiungere una diagnosi e soprattutto una diagnosi univoca? [4]. Vi è una relazione additiva tra le differenti fonti di informazioni, o esiste invece una relazione moltiplicativa tale per cui l'interpretazione di un rischio sia in realtà la variabile di un altro rischio?

Lincertezza è anche legata al fatto che uno stesso dato assume significati diversi in contesti differenti: una temperatura corporea elevata, ad esempio, può essere interpretata in modi diversi a seconda delle condizioni generali del paziente. Inoltre, i processi di cura richiedono spesso valutazioni, e quindi decisioni, multiple, esponendo di conseguenza i pazienti a rischi multipli [5].

Gli avanzamenti della scienza e delle tecnologie hanno portato a due conseguenze, in particolare nell'ambito delle cure delle patologie croniche: da un lato i pazienti, grazie a nuove possibilità terapeutiche, hanno visto aumentare la propria prospettiva di vita. Dall'altro i malati, sempre più esperti e aggiornati nel campo dei loro problemi di salute, accumulano informazioni che possono guidare le loro decisioni relative al modo con cui vorrebbero convivere con la patologia cronica e al modo in cui intendono la loro qualità di vita.
Questi soggetti devono prendere numerose decisioni multiple e ripetitive, i cui esiti non sono sempre prevedibili al momento della scelta.

Ad esempio, nel caso dell'artrite reumatoide, patologia che tende a cronicizzare e quindi ad accompagnare il paziente per tutta la vita, la gestione comporta non una decisione singola, ma una serie di decisioni prese nel tempo, ciascuna delle quali deve essere concordata tra medico e paziente. Inoltre, come spesso avviene nella cura di una patologia e delle sue complicanze, la gestione coinvolge più medici con diverse specializzazioni (in questo caso, reumatologo, medico di base, ortopedico), oltre che professionisti esperti nei processi di cura, e di conseguenza anche da questo punto di vista nelle decisioni sono coinvolti più soggetti.

Un altro esempio di complessità decisionale è fornito dalla cura del diabete mellito, una malattia cronica nella quale l'obiettivo terapeutico consiste nel mantenere stabile la glicemia. Per ottenere questo risultato, è necessario che il paziente esegua un esercizio fisico regolare e rispetti una dieta adeguata. La mancanza di esercizio e la scarsa aderenza alla dieta aggravano le condizioni del malato e possono favorire la comparsa di complicanze. Allora, in che modo un medico deve presentare al proprio assistito le informazioni se vuole che il paziente diabetico sia realmente consapevole dei rischi connessi alla sua malattia e possa quindi prendere delle decisioni informate su come adottare e mantenere uno stile di vita in grado di ridurre o procrastinare le complicanze della sua malattia?

La percezione dei livelli di rischio deve venire acquisita dal paziente in un determinato contesto, e, se possibile, con l'aiuto e l'assistenza di un professionista. Va ricordato che l'impatto delle decisioni del paziente può non essere sempre prevedibile $\mathrm{e}$ che le sue scelte possono essere arbitrarie.

A questo punto bisogna porsi delle domande:

- quando vi sono incertezze relative alla diagnosi e alla terapia, quali sono i livelli di rischio accettabili dal paziente e quali sono i livelli di rischio accettabili dal medico?

- come fanno il medico e il paziente a valutare l'impatto di opzioni di cura diverse?

- come fanno il medico e il paziente a definire l'impatto di tutte le variabili possibili?

I fattori psicologici, come ad esempio l'atteggiamento del paziente riguardo ai rischi (tenendo conto che il rischio personale si riferisce a una persona singola), hanno influenza sulla percezione del rischio da parte del malato. Anche le politiche sanitarie hanno un ruolo importante nel campo della percezione del rischio da parte del paziente.

In una situazione definita, nella quale è necessario prendere una decisione clinica, la percezione del rischio per il paziente può essere specifica, men- 
tre la percezione del medico è spesso quella del rischio in generale. In determinate situazioni i pazienti possono essere anche più prudenti dei medici per quanto riguarda la percezione del rischio, in quanto i risultati delle cure interessano di più ai malati, che vi sono coinvolti in prima persona, che non al medico.

D'altro canto la stima del rischio è soggettiva: è noto che vi sono individui portati a sovrastimare il rischio connesso a comportamenti non salutari, anche rispetto a ciò che viene loro descritto dal medico, mentre altri minimizzano le conseguenze. Le persone tendenzialmente ottimiste tendono a sottostimare i rischi personali che corrono, pur conoscendo il concetto di rischio in generale. Risulta quindi evidente che medici e pazienti hanno spesso differenti punti di vista quando si tratta della valutazione del rischio. Il problema a questo punto può essere risolto mediante una decisione condivisa, il che però porta a soluzioni non sempre scevre di complicazioni.

\section{DISCUTERE SUI RISCHI E LE INCERTEZZE}

Il medico, per fornire cure efficaci, deve acquisire e capire la prospettiva del paziente [6]. Purtroppo da un lato vi è scarsezza di linee guida basate sulle evidenze che supportino la decisione clinica, e dall'altro mancano indicazioni relative a come le evidenze scientifiche vadano spiegate ai pazienti, aiutandoli a prendere le decisioni più utili per se stessi, e infine non vi sono linee guida che insegnino come condurre una discussione bilanciata riguardo a rischi e incertezze.

Solo pochi medici sono a conoscenza del cosiddetto "effetto cornice" che entra spesso in gioco quando si discute di rischi con il paziente. L'effetto cornice è definibile come la percezione soggettiva del decisore relativa ai risultati associati con una particolare scelta. Il contesto nel quale il decisore opera le sue scelte può essere influenzato non solo dalle idiosincrasie del decisore stesso, ma anche dalla modalità con la quale vengono spiegate al paziente le probabilità di incorrere in un determinato rischio.

L'effetto cornice è definibile anche come la modalità con la quale vengono presentate le varie opzioni decisionali, le quali possono variare a seconda del modo di formulare il problema. Un esempio di questa variazione si può osservare quando il beneficio viene considerato in termini di riduzione relativa del rischio (RRR) o in condizioni di riduzione assoluta del rischio (ARR). Uno studio, eseguito su 700 donne australiane con lo scopo di verificare se fossero d'accordo sullo screening mammografico fornito dallo Stato, ha permesso di verificare che le risposte erano diverse a seconda di come venivano presentati i risultati: se il beneficio era espresso in termini di RRR, la maggior parte delle intervistate ha risposto che vi avrebbe aderito, al contrario di come accadeva se era espresso in ARR [7].

In un altro studio, a 3.201 danesi sani venne richiesto se preferivano, per ridurre i rischi connessi con le decisioni mediche, avere informazioni sull'ARR o sull'RRR. La scelta fu quella del RRR [8].

Le preferenze delle persone relative alla riduzione dei rischi connessi con gli interventi sanitari dipendono anche dal singolo individuo e dal contesto nel quale vengono proposte.

Le modalità di presentazione dei rischi sono anch'esse importanti per l'accettazione o meno degli interventi proposti. Ad esempio, si ha o non si ha un "effetto cornice" a seconda di come vengono presentate le informazioni relative ai rischi, e in particolare se le informazioni vengono presentate come un guadagno (200 persone su 600 sopravvivono) o come una perdita (400 persone su 600 non sopravvivono). Le informazioni relative a un intervento presentate come un vantaggio ispirano una reazione di evitamento, mentre se le informazioni vengono presentate come una perdita, tendono a indurre un comportamento che minimizza il peso del rischio.

Armstrong ha scoperto che definire la possibilità di morte nel tempo determina livelli inferiori di comprensione e meno interesse nella chirurgia preventiva, mentre il contestualizzare la situazione in termini di sopravvivenza fa sì che le persone accettino più facilmente gli interventi proposti [9].

Queste considerazioni rendono chiaro che le decisioni hanno le loro radici in un contesto nel quale sono presenti molti altri fattori, contesto nel quale la prospettiva psicologica può variare.

Il singolo individuo può considerare le cure come una perdita, specialmente se le cure sono legate a una malattia, mentre la convalescenza viene considerata come un guadagno. In altre parole, il deterioramento dovuto alla malattia rappresenta una perdita, mentre la guarigione da una malattia viene considerata un vantaggio.

Un fattore addizionale (dalla parte della perdita) è dato dal rischio degli effetti collaterali da farmaci. Il rischio dell'insorgenza di effetti collaterali può avere una maggiore influenza sulla scelta del trattamento da parte del paziente di quanto non ne abbia per il medico che è più incline a verificare il successo che non gli effetti collaterali del trattamento prescritto. Una persona con artrite reumatoide cronica è costretta a curarsi per tutta la vita con farmaci come il metotrexate, che ha un buon effetto terapeutico, ma che può causare effetti collaterali.

In conclusione bisogna accettare che i rischi vengano vissuti diversamente a seconda dei risultati 
proposti od ottenuti, i quali definiscono se il trattamento è stato un successo o un insuccesso.

Un'altra possibile fonte di discrepanze nei giudizi e nelle scelte di trattamento può essere costituita dal modo con il quale vengono presentate le varie opzioni terapeutiche, le quali possono andare dalla possibilità di avere risultati scarsi (con una bassa probabilità) alla possibilità di avere risultati buoni (con relativamente alta probabilità).

I clinici che discutono i rischi delle varie opzioni di intervento con i pazienti devono tenere in conto sia la gravità che la frequenza dei rischi dei trattamenti proposti.

Un medico che prescrive un test di screening a un paziente deve per forza considerare il fatto che, mentre il rischio che il paziente sia positivo per una particolare malattia è piccolo, nel caso di un eventuale errore nei processi di diagnostica, una falsa negatività avrebbe un impatto notevole in quanto le conseguenze potrebbero essere importanti. Per un medico che prende in considerazione il rischio di errori nella diagnostica, il giudizio si basa sui dati esistenti, per cui il medico può fare diverse considerazioni relative al rischio di un paziente singolo. Il paziente, da parte sua, non è a conoscenza che esistono questi dati, in quanto le informazioni sulla sua patologia gli vengono fornite dal medico.

Altri fattori che determinano la percezione del rischio sono costituiti dal negare il rischio: un esempio di questa tendenza è che la maggior parte dei fumatori non si considera a rischio di cardiopatia o di neoplasia.

\section{SUPERARE IL DIVARIO TRA CLINICI E PAZIENTI}

La convinzioni relative alla salute e la percezione dei rischi si sono dimostrati essere uno dei maggiori fattori che influenzano le decisioni relative al trattamento delle malattie. Il livello di rischio percepito varia a seconda delle diverse patologie e dal loro livello di gravità. Mentre il medico ha una conoscenza generale del rischio legato alle malattie specifiche, il paziente non possiede queste conoscenze.

Medici e pazienti spesso non sono d'accordo sulle preferenze relative al trattamento, il che rende difficile la presa di decisioni. Infatti molte volte non vi è accordo in merito alla scelta delle opzioni terapeutiche migliori e sulla maggiore o minore rischiosità delle varie opzioni disponibili. Differire nel tempo la valutazione del rischio può potenzialmente vanificare gli effetti della terapia proposta dal medico.
Questo tema è spesso rilevante nel caso dell'artrite reumatoide, una malattia progressivamente invalidante, nella quale vanno considerate attentamente le variabili delle decisioni da prendere. In questo caso, le decisioni dipendono da quanto il paziente da una parte e il medico dall'altra diano valore alle scelte terapeutiche. Quale valore danno il medico e il paziente alle variabili come il dolore e la disabilità nel caso di una lunga durata della malattia? Forse, per il paziente, il dolore rappresenta il disturbo più importante, mentre per il medico è più importante valutare nel tempo l'andamento della disabilità, nel caso il paziente abbia una sopravvivenza lunga. Queste considerazioni hanno necessariamente un impatto importante sulle scelte terapeutiche.

Alcuni pensano che una piccola differenza nella percezione del rischio tra medico e paziente non sia rilevante, per cui molti tendono a una soluzione concordata tra medico e paziente. Va ricordato tuttavia che ridurre la distanza delle posizioni tra medico e paziente richiede una notevole elasticità e disponibilità da parte del clinico. Quest'ultimo, infatti, deve osservare i comportamenti del paziente in relazione alla malattia in quanto l'evoluzione nel tempo dei comportamenti del paziente segnala una maggiore consapevolezza della malattia e dei possibili rimedi.

\section{CONCLUSIONI}

Da tempo si sta sviluppando un interesse sempre maggiore sul coinvolgimento del paziente nelle decisioni sanitarie che lo riguardano. Le preferenze e le opinioni dei pazienti devono essere prese in considerazione quando i medici definiscono iltrattamento.

Purtroppo in questo campo non solo le evidenze sono limitate, ma gli stessi pazienti non sono in grado di scegliere tra le varie opzioni di cura. In questo contesto è difficile decidere quali siano le informazioni sanitarie che devono essere fornite al paziente. Il processo di valutazione dei rischi conseguenti può essere difficile a causa di incertezze e di difficoltà di scelta che si manifestano nel corso dell'analisi del quadro clinico, della definizione della diagnosi e della progettazione della terapia. I pazienti che sono stati informati in modo adeguato e corretto sono più disposti a partecipare attivamente al processo di cura, mostrano maggiore soddisfazione rispetto al trattamento proposto, con conseguenti risultati migliori in termini di qualità ed efficacia delle cure.

Sebbene sia riconosciuto che tenere in giusta considerazione il punto di vista del paziente nei processi di cura sia utile per ottenere il consenso alla 


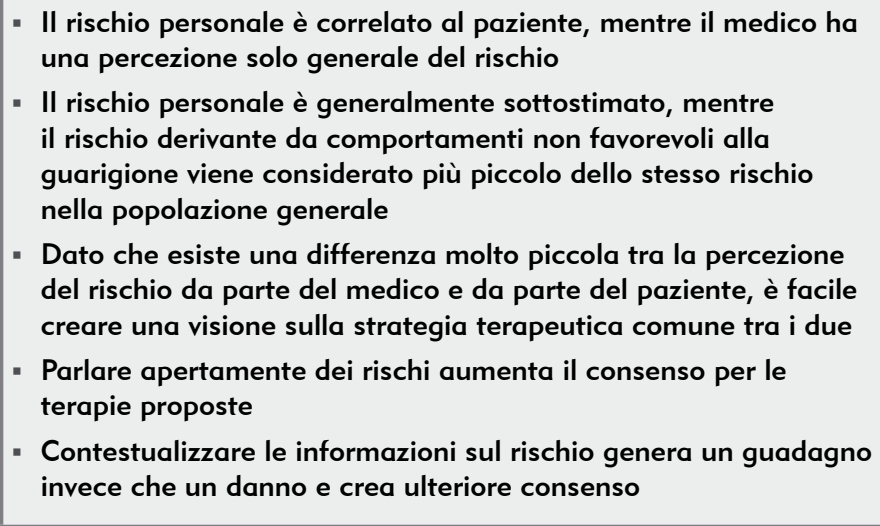

Tabella I. La valutazione del rischio nella pratica medica cura stessa, mancano al momento delle linee guida basate sulle evidenze che indirizzino il medico nella fase di discussione e condivisione dei processi di cura con il proprio assistito.

La mutua collaborazione tra medici e pazienti fa inoltre sì che, per il clinico, diventi possibile esplicitare ai pazienti il concetto che il rischio non può essere totalmente eliminato nell'attività clinica. I giudizi e le decisioni sul come ridurre il rischio sono complicate, così come i giudizi (Tabella I). Le decisioni terapeutiche non sono semplici, ed è necessario che le cure evolvano dall'uso di regole generali all'uso di regole più specifiche, come potrebbe essere ad esempio la conoscenza in migliore dettaglio dei fattori di contesto.

\section{BIBLIOGRAFIA}

1. Endozien LC. Thinking in numbers and using numbers to communicate risks. Clinical Risk 2007; 13: 1-2

2. Gigerenzer G. Reckoning with risk: learning to live with uncertainity. London: Penguin Books, 2002

3. Orthendahl M. Framing risk in clinical practice. Clinical Risk 2007; 13: 53-5

4. Watt S. Clinical decision-making in the context of chronic illnesses. Health Expect 2000; 3: 6-16

5. Parmigiani G. Uncertainty and the value of diagnostic information, with applications to axillary limph node dissection in breast cancer. Stat Med 2004; 15: 843-55

6. Slovic P. The perception of risk. London: Earthscan, 2000

7. Young JM, Davey C, Ward JE. Influence of the "Framing Effect" on women's support for government funding of breast cancer screening. Aust N ZJ Public Health 2003; 27: 287-90

8. Gyrd-Hansen D, Kristiansen IS, Nexoe J, Nielsen JB. Effects on baseline risk information on social end individual choices. Med Decis Making 2002; 22: 71-5

9. Armstrong K, Schwartz JS, Fitzgerald G, Putt M, Ubel PA. Effect of framing as gain versus loss on understanding and hypotetical treatment choices: survival and mortality curves. Med Decis Making 2002; 22: 76-83

\section{CORRESPONDING AUTHOR}

Prof. Giulio Marcon, e-mail: giulio.marcon@infinito.it 